\title{
Detection and Classification of Pregnancy StateUsing Deep Learning Technique
}

Hassan Abdelrhman Mohammed ${ }^{1}$, .Eltahir Mohmmed Hussein ${ }^{2}$, Mahir Mohammed Sharif ${ }^{3}$ 1Dept. of Computer Sciences Faculty of Computer Sciences \& Information Technology, Karary University, Khartoum,Sudan

2Dept of Electrical and Electronic Engineering, Faculty of Engineering Sudan University of Science \&Technology,Khartoum, Sudan.

3Dept. of Computer Science Faculty of Computer Science \& Information Technology, Omdurman Islamic University, Omdurman, Sudan

Corresponding author

Mahir Mohammed Sharif, Email: mahiralsharif@yahoo.com

\section{To cite this article:}

Hassan Abdelrhman Mohammed, .Eltahir Mohmmed Hussein, Mahir Mohammed Sharif, Detection and Classification of Pregnancy StateUsing Deep Learning Technique, Omdurman Islamic University Journal,Vol. 17, No. 2, 2021, pp $71-85$ • https://doi.org/10.52981/oiuj.v17i2.1819

Received: 14/9, 2021; Accepted: 24/11, 2021; Published: 28/ 12, 2021.

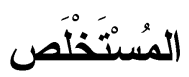

الهدف من هذا العمل هو تصميم وتطوير نموذج يكنشف ويصنف الحالة الصحية للحمل .الموجات فوق الصوتية هي و احدة من أكثر تطور ات التصوير السريري انتشارًا، حيث تمكّن الطبيب من تقييم الأمراض وتحليلها وعلاجها. تودي معظم مضاعفات الحمل إلى مشاكل خطيرة تقيد النمو السليم الذي يسبب الضعف أو الوفاة. في هذا العمل تم تطوير نموذج معالجة الصور للتعرف على صحة الجنين أثناء الحمل وتصنيفه لجميع مر احل نموه. تم تتفيذ التقنيات في التعلم العميق حيث تم تطبيق نموذج)CNN (Resnet50)للتعرف على الصور لاكتشاف وتصنيف الحالة الصحية للجنين من صور الموجات فوق الصونية. النموذج المقترح ساهم بتقديم حل منكامل لكل فترة الحمل يعمل على التعرف على كل مر احل نمو الجنين ابتداء من مرحلة ما قبل الحمل (وهنا يتعرف على صلاحية الرحم للحمل وحجم البويضة وقابليتها لتكوين الجنين) وحتى مرحلة الو لادة وذلك من خلال التدريب و التحقق والاختبار باستخدام تقنية التحقق المتقاطع التي استخدمت فيها خمس طيات لدفة التشخيص وذلك وفقا للأنماط التي تميز كل مرحلة عن الأخرى و التحقق من انه سليم او غير سليم في المرحلة المعنية. عززت هذه الدراسة دقة التشخيص باستخدام نقل التعليم و استخدامه للصور المدخلة الجديدة التي لم يتم التدريب عليها كتغذية عكسية.حقق النظام دقة بلغت نسبة 96.5 


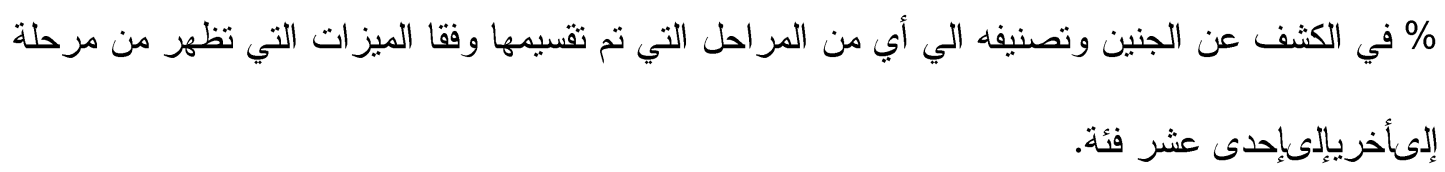

\begin{abstract}
This work aims to design and develop a model that detects and classifies pregnancy health status. Ultrasound is one of the most prevalent developments in clinical imaging, as it enables a doctor to evaluate, analyze and treat diseases. Most complications from pregnancy lead to serious problems that restrict healthy growth, causing weakness or death. In this work, an image processing system was developed to recognize the health during pregnancy and classify it for all stages of its development. The technique in deep learning has been implemented, as CNN (Resnet50) image recognition model was applied to detect and classify fetal health status from ultrasound images. The proposed model contributed to providing an integrated solution for each pregnancy period that works to identify all stages of fetal development, starting from the pre-pregnancy stage (here it is known about the suitability of the uterus for pregnancy, the size of the ovum, and its ability to form the fetus) and up to the stage of birth, through training, verification and testing using the crossverification technique that five folds of the diagnostic rudder were used under the patterns that distinguish each stage from the other and to verify that it is sound or unsound in the concerning stage. This study enhanced diagnostic accuracy by using transfer learning and novel accessory images that were not trained as feedback. The model achieved an accuracy of $96.5 \%$ in detecting the fetus and classifying it into any of the stages that were divided according to the features that appear from one stage to the next to eleven categories.
\end{abstract}

Keywords-UltrasoundImage, Recognition, Fetusdetection, Deep Learning \&CNN.

\title{
I. Introduction
}

Pregnancy is a stage during which babies create inside a woman. Fetal health is the sign of proper growth of the embryo in the pregnant woman's uterus during the incubation period (Deressa \& Kadam, 2018). The quantity of ladies who face a high-hazard pregnancy is expanding because of the deferred marriage and childbearing. A fetus's growth can be affected by pregnancy confusion genuinely, which will probably lead to helpless pregnancy results(Wang et al., 2018).Ultrasound (US) imaging is a safe non-intrusive system for diagnosing inner body organs. Ultrasound imaging when contrasted with other imaging apparatuses, for example, computed tomography(CT) and magnetic resonance imaging (MRI), is less expensive, versatile and more pervasive. It assists with diagnosing the reasons for torment, growing, and contamination in inside organs, for assessment and 

and Classification of Pregnancy StateUsing Deep Learning Technique,

therapy of ailments. Ultrasound imaging has transformed into an overall exam strategy for pre-birth conclusion. It is utilized to explore and quantify fetal biometric boundaries, for example, the fetu's stomach periphery, head outline, biparietal measurement, femur and humerus length, and crown-rear end length(Ishikawa, Xu, Ohya, \& Iwata, 2019). Besides, the fetal head circumference (HC) is estimated for assessing the gestational age, size and weight, development checking and distinguishing embryo variations from the norm (Sobhaninia et al., 2019).The aim of this work is to develop a detection and classification system to accurately detected fetus state during pregnancy period which present in ultrasound images, and to differentiate thenine periods.In input layer stages digital image processing techniques are used to obtain high-quality medical images.

\section{II.Literature Review}

Various techniques for the deep learning are proposed in literature.Omneya Attallah and etc. in(Attallah, Sharkas, \& Gadelkarim, 2020) proposed a new framework based on deep learning algorithms is proposed for detecting ENDs.This study solved due to several steps, beginning from imaging of the undeveloped brain cerebrum and preparing such sorts of images, and finishing with segmenting the brain, identifying the irregularity, and characterizing it. segmenting undeveloped brain from MRI images is fundamentally more difficult than fragmenting grown-up MRI cerebrum images. This study used a system that initially utilizes the crude images to develop three profound convolution neural networks (DCNNs) of different structures to identify ENDs. Subsequently, it removes profound highlights from the three DCNNs to fabricate three support vector machine classifiers individuallytrained with each deep feature of the three networks.

Tran, Cooke, Illingworth, \& Gardner, (2019)proposed a method for detection of pregnant The deep learning model was trained using time-lapse videos with known FH pregnancy results to play out a parallel order undertaking of anticipating the likelihood of pregnancy with FH given time-slip by video grouping. The prescient intensity of the model was estimated utilizing the average area under the curve(AUC) of the beneficiary operating characteristic bend more than 5-crease defined cross-approval. The profound learning model had the option to foresee FH pregnancy from time-lapse by recordings with an AUC of 0.93 [95\% CI 0.92-0.94] in 5-overlay defined cross-approval. A hold-out approval test across eight research centers demonstrated that the AUC was reproducible, going from 0.95 to 0.90 across various labs with various cultures and lab measures.

Mu, Feng, Yang, \& Wang, (2018) proposed a clever plan to recognize In this work by buildup a deep learning algorithm which can identify and arrange unfavorable pregnancy results before parents getting pregnant. We train a multi-layer neural network by utilizing a dataset of 75542 couples' multi-measurement pre-pregnancy wellbeing information. Our model beats some of the algorithms in accuracy.

(Rawat, Jain, \& Shrimali, 2018)this study a definite has been offered on fetal biometric boundaries and nuchal clarity to feature the examination. approaches with a level of approval in assorted clinical areas. At that point, a classification of the bibliographic 
appraisal of the segmentation field of ultrasound 2D fetal images has been introduced. The fetal images of high-hazard pregnant women have been taken into the daily schedule and ceaseless observing of fetal boundaries. These boundaries are utilized.

the study (Zhang, Petitjean, Yger, \& Ainouz, 2020) researched different saliency map techniques to use their mastery to tackle CNN's anticipated worth relapse. Since saliency maps techniques have been produced for arrangement $\mathrm{CNN}$ generally deciphers relapse saliency guides and development of a bother based quantitative assessment of proof strategies. Results got on a public dataset of ultrasound pictures show. Some saliency maps show the head shape as the most applicable highlights to survey the head circuit. The guide quality relies upon the spine structure and whether the forecast blunder is low or high.

The study in (Luo, Liu, Li, \& Zhang, 2019) proposed a programmed picture quality appraisal plot dependent on perform multiple tasks figuring out how to aid FS picture quality control. A basic rule for FS picture quality control is that all the essential anatomical structures in the part ought to show up full and striking with a reasonable limit. Henceforth, our plan intends to distinguish those fundamental anatomical structures to decide whether a FS picture is the standard picture accomplished by three convolutional neural organizations. The Feature Extraction Network means to separate profound level highlights of FS pictures. The Class Prediction Network chooses whether the structure fulfills the guideline, and Region Proposal Network recognizes its area dependent on the separated highlights. The plan has been applied to three sorts of fetal segments: head, stomach, and heart. The test results show that our strategy can make a quality appraisal of a FS picture inside not exactly a second. Likewise, our strategy accomplishes serious execution in both the identification and characterization contrasted and best in class procedures.

Paper in (Al-Bander, Alzahrani, Alzahrani, Williams, \& Zheng, 2019) proposed a profound learning-based strategy to portion fetal head from ultrasound images. The proposed strategy details the discovery of the fetal head limit as the consolidated item confinement and division issue dependent on a profound learning model. Fusing an article limitation in a structure created for division purposes plans to improve the division exactness accomplished by a completely convolutional network. At long last, an oval is fitted on the shape of the divided fetal head utilizing the least-squares oval fitting technique. The proposed model is prepared on 999 2-dimensional ultrasound pictures and tried on 335 

and Classification of Pregnancy StateUsing Deep Learning Technique,

pictures accomplishing a Dice coefficient of 97.73 \pm 1.32 . The exploratory outcomes exhibit that the proposed profound learning strategy is promising in programmed fetal head recognition and segmentation.

The study in (Sobhaninia, Emami, Karimi, \& Samavi, 2020) explored the effects of utilizing multi-scale contributions to the organization. additionally, proposed a light convolutional neural organization for programmed $\mathrm{HC}$ assessment. Test results on a ultrasound dataset of the hatchling in various trimesters of pregnancy show that the division exactness and $\mathrm{HC}$ assessments directed by a light convolutional neural organization are like profound convolutional neural organizations. The proposed network has a few boundaries and requires a more limited preparing time.

Authors in (Baumgartner et al., 2017)proposed a novel strategy dependent on convolutional neural organizations that can consequently identify 13 fetal standard perspectives in freehand 2D ultrasound information just as give confinement of the fetal structures by means of a bounding box. A significant commitment is that the organization figures out how to restrict the objective life structures utilizing powerless management dependent on picture level marks as it were. The organization engineering is intended to work progressively while giving ideal yield to the restriction task. We present outcomes for ongoing comment, review outline recovery from saved recordings, and restriction on an extremely huge and testing dataset comprising of pictures and video chronicles of full clinical irregularity screenings. We found that the proposed strategy accomplished a normal F1-score of 0.798 in a sensible grouping test demonstrating continuous identification, and got a $90.09 \%$ precision for review outline recovery. Additionally, the precision of $77.8 \%$ was accomplished on the restriction task.

The study in (Grandjean et al., 2019) used 225 fetus will be estimated at 16-30 weeks of growth. For every hatchling, six volumes (two for the head, mid-region, and thigh, separately) will be tentatively obtained in the wake of performing standard 2D biometry estimations (head and stomach circuit, femoral length). Every volume will be prepared later by both a product and an administrator to remove the reference planes and to play out the relating estimations. The various arrangements of estimations will be contrasted utilizing Bland-Altman plots with survey the understanding between the various cycles (I). 
The attainability of utilizing the product in clinical practice will be surveyed through the disappointing pace of preparing and the score of nature of estimations (II). Interclass relationship coefficients will be utilized to assess the intra observer and between spectator reproducibility (III).

Through investigation in the previous studies, it was found that there is a match in the use of the type of data, which is (ultrasound images), and also that all studies used deep learning technology, but many of them used different models and approaches, and also differed in the target identified in the ultrasound images as most The studies cover a part of the fetus such as the head, limbs, chest and gender, etc. Some of them also studied a specific period of pregnancy, such as the first trimester only, or the second or third trimester. This study was distinguished by providing an integrated solution that included the pre-pregnancy stage and the whole life of the fetus during the pregnancy period from the first week until birth.

\section{II.MATEERIALS AND METHOD}

System architecture used for fetal detection during pregnancy involves the following main steps: an image input layer, convolution layer, pooling layer, fully connected layer, fetus classification, and output layer. The block diagram shown in Fig 1 illustrates all system steps. Matlab is used in all system implementation steps.

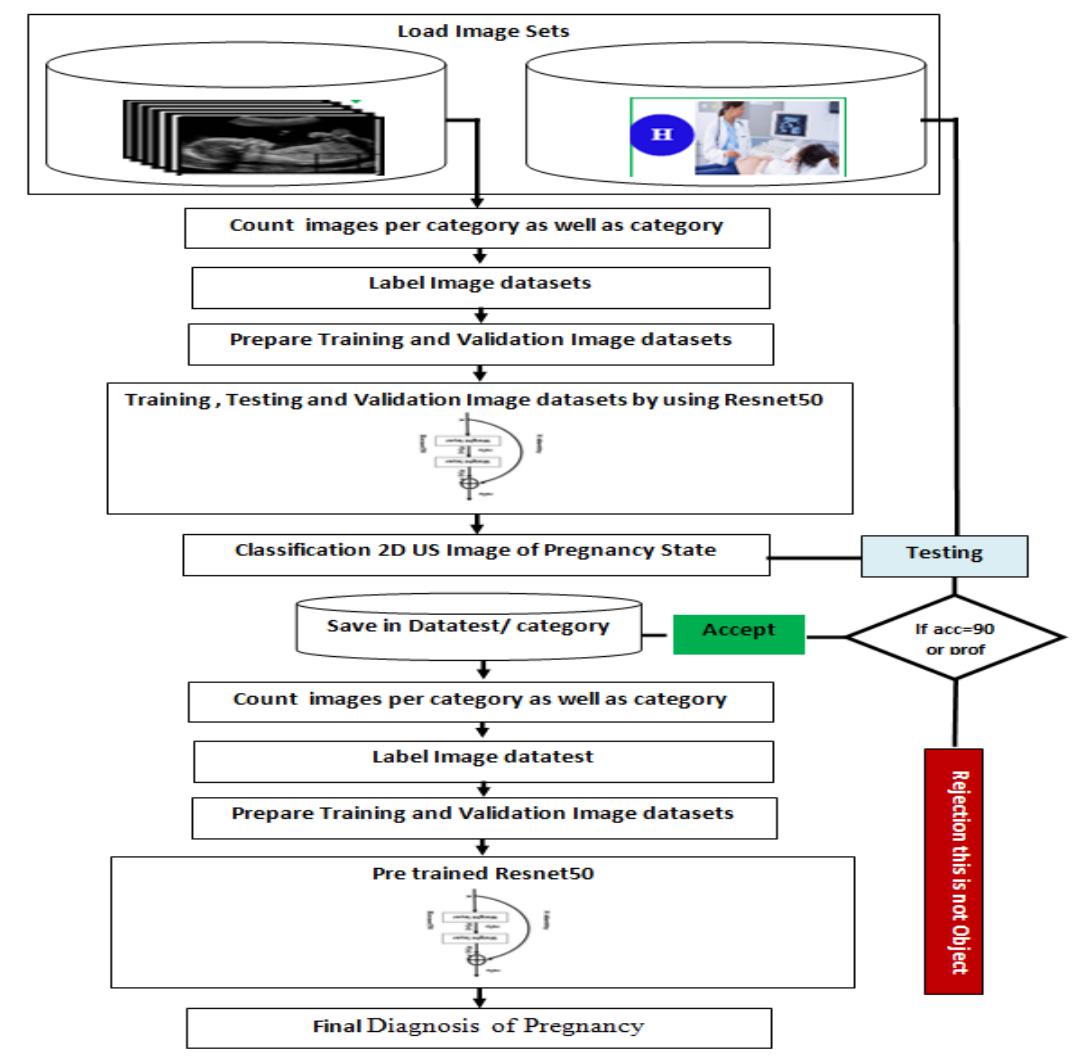



and Classification of Pregnancy StateUsing Deep Learning Technique,

\section{A. Deep Learning \& CNN}

Deep Learning is a particular machine learning subfield, in new learning portrayal of information underlining "consecutive" layer learning of description. Deep Learning licenses computational models organized in numerous readiness layers to examine report spoke to by the remarkable degree of abstraction(Tahir, Badriyah, \& Syarif, 2018). Deeplearning innovation for AI has pulled in incredible consideration as it continually breaks records in an assortment of necessary benchmark tests. As one kind of technique that fulfills the prerequisites of deep learning, $\mathrm{CNN}$ is presently a cutting edge profound learning structure applied to a broad scope of fields, for example, programmed machine interpretation, PC vision, and speech recognition. Incredible execution in object location or grouping from images has been demonstrated because CNN networks give a quicker, more vigorous, and more advantageous calculation than traditional neural networks. In $\mathrm{CNN}$, image pixels could be straightforwardly utilized as a contribution to the standard feedforward neural networks. Although many pixels from even little image patches bring about an enormous number of association weight boundaries to be prepared, CNN models consolidate loads into a lot more modest bit channels that drastically rearrange the learning model(Qu, Xu, Ding, Jia, \& Sun, 2019).In clinical imaging, the most ordinarily utilized deep learning strategies are convolutional neural networks (CNN) Compared features inside the information itself. By and large, $\mathrm{CNN}$ are preferable ready to distinguish features over the human eye(Diniz, 2020).

Deeplearning techniques have been used for ultrasound image analysis in pregnancy to address such tasks as classification, object detection, and tissue segmentation. This review covers applications in pregnancy. Matlab CNN functions are used for implementation of the following steps: input dataset of US images fetus for all nine period show in table 1 followed, feature extraction by applied convolution layer technique, max pooling layer and fully connected layer techniques to detection and classification the fetus health states.

\section{B. Image Dataset}

The kaggle ultrasound images dataset is the source of information required for the analysis and development of fetal health detection and classification model. US images information also provides a visualized unhealthiness of the fetus that helps for early intervention before the risk happening. total of 2,200 instances with 11 chosen attributes which are multivariate datatypes divided to 11 classing base on fetal growing start from pre pregnancy, 1-8week, 9 - 12week, ...,37 - 40week and abnormal pregnancy are shown in Table 1. 
TABLE1 ELEVEN PERIOD OF FETAL GROWTH

\begin{tabular}{|c|c|c|}
\hline $\begin{array}{l}\text { Periods/we } \\
\text { ek }\end{array}$ & Descriptions & Changes \\
\hline $\begin{array}{l}\text { Prepregnanc } \\
\mathrm{y}\end{array}$ & Size $<18$ & \\
\hline $1-8$ weeks & $\begin{array}{l}\text { Placenta, brain, tissues, heart, buds, } \\
\text { intestines, eyes, ears, nose }\end{array}$ & Begin to form and develop \\
\hline 9-12 weeks & $\begin{array}{c}\text { Teeth , fingers, bones, muscles, backbone, } \\
\text { hands,arm }\end{array}$ & arm \& hand long than feet \& legs \\
\hline 13-16 weeks & $\begin{array}{l}\text { external sex organs, outer ear swallow, neck } \\
\text { and kidneys. }\end{array}$ & Begin to form and develop \\
\hline 17-20 weeks & Sucking, reflex, nails, .. & Develop and more active \\
\hline 21-24 weeks & Fetus may hiccup, tear ducts and lungs & Appear \& developing \\
\hline $25-28$ weeks & $\begin{array}{l}\text { Eyes can be open \& close, fetus kicks, fetus } \\
\text { responds to sound }\end{array}$ & fetus responds to sound \\
\hline 29-32 weeks & $\begin{array}{l}\text { Bones harden, hair and langue begins to } \\
\text { disappear }\end{array}$ & begins to disappear \\
\hline 33-36 weeks & Fetus development finished & Turn in to head- down position \\
\hline 37-40 weeks & Fetus more fat accumulates & Drops \& lower in to pelvis \\
\hline
\end{tabular}

\section{Pre-processing}

Used in this study a mini-batch size of 512 with 8 GPUs for training our models. The initial learning rate is set to 0.01 . We train with a total number of 100 epochs, and the learning rate is multiplied by 0.1 at five epochs. The weight decay is not used (set to 0 ) for training and applied this strategy to all proposed experiments - architectures we choose.

Used the create a new model for ultrasound images dataset is divided to 11 folders as datastore according to feature which appears of fetal growing during pregnancy age periods see table 4.1 ultrasound image of a fetus was resized to $224 \times 224$. Of the total 2,200 images selected from the dataset used in this study, cross-validation is set to 5 as the value of folds, which means two-third were used for training, while the other One third was used for validation. Hence, 1760 images were used to train the network, and 440 images were used for validation This is shown in Fig 2.

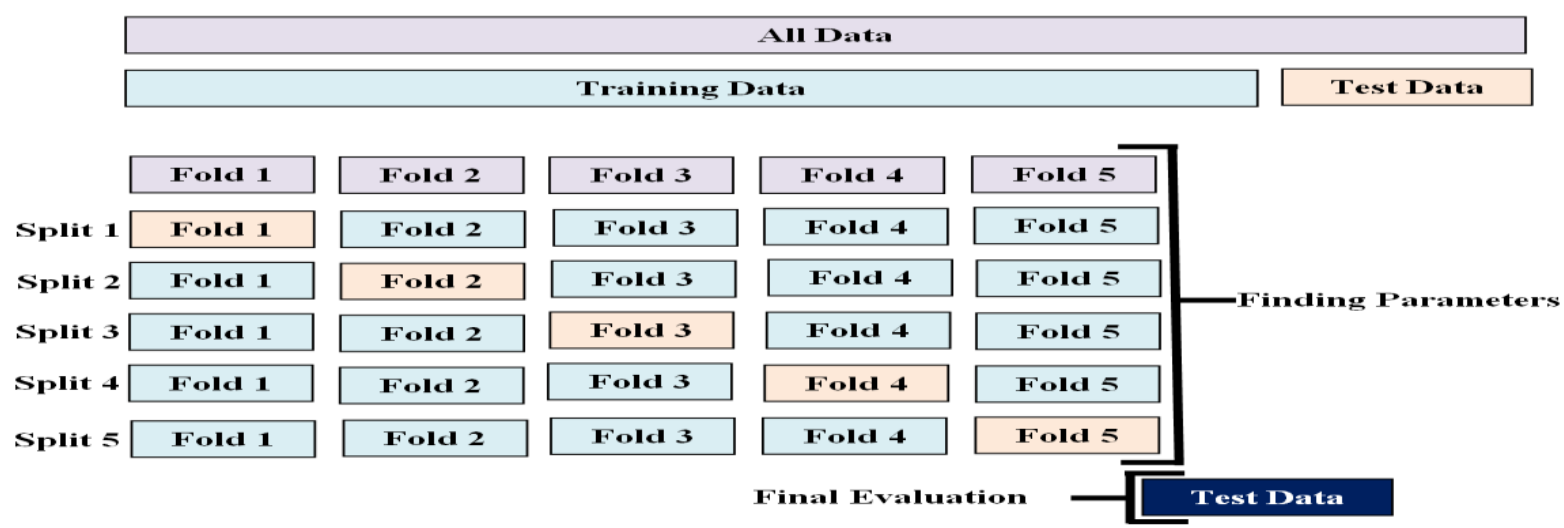

Figure 2: cross-validation for image datastore 


\section{EXPERIMENTS}

The experiment was conducted using Matlab version R2018b program in all stages of the investigation from preparation to reaching the results, which can be described as follows:

\section{D. labeled and adjust number of images in datastore}

Start the proposed Experiment by Create an Image Datastore to help you manage the data. Because Image Datastore operates on image file locations, images are not loaded into memory until reading, making it efficient for extensive image collections. This step is significant, so I proposed a labeled base on fetal growth divided into 11 categories. Each one contends 100 ultrasound images for different fetus stages during pregnancy and abnormal, so this stage contains the images and the category labels associated with each image. The labels are automatically assigned from the folder names of the image files. And summarize the number of images per category show in Table2.

TABLE 2 CATEGORY LABELS ACCORDING TO FETUS GROWING

\begin{tabular}{|l|c|}
\hline Label & Count \\
\hline Ready_to_pregnancy & 200 \\
\hline from1_8weeks & 200 \\
\hline from9_12weeks & 200 \\
\hline from13_16weeks & 200 \\
\hline from17_20weeks & 200 \\
\hline from21_24weeks & 200 \\
\hline from25_28weeks & 200 \\
\hline from29_32weeks & 200 \\
\hline from33_36weeks & 200 \\
\hline from37_40weeks & 200 \\
\hline Abnormal & 200 \\
\hline
\end{tabular}

In the situation, obtained new ultrasound image from the proposed system's feedback that makes image data Datastore an unequal number of images for each category. Let's first adjust it so that the number of images in the training set is balanced using the MATLAB function. And select an image for each file labeled to make sure. Fig 3 Below a presented example of images from 11 of the categories included in the dataset. 

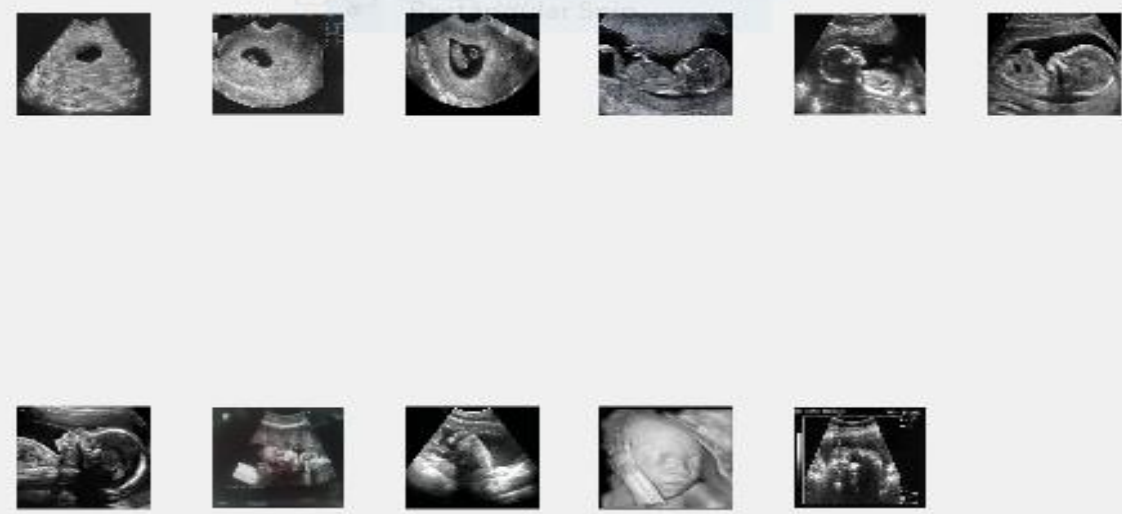

Figure 3: Selected image from each file labeled of midstto make sure the system is ready for training

\section{E. Training and Fine-Tuning for Detection and Classification Fetus:}

Convolutional neural networks automatically learn the best deputy features from the fresh data to extract feature In. This study used the ResNet50 CNN for the proposed system method due to its state-of-the-art performance in fetal detection in the challenging, complex detection and classification of the fetus during pregnancy. We modified the input layer to fit the size of the extracted image objects. Our pre-training on the fetal ultrasound image patch dataset involved finding the set of CNN layer weights and used a crossvalidation model with three folds.

\section{F. Data Augmentation}

Due to the move in the position of the fetus inside the woman's womb. This proposed system was used an image data augmenter that configures a set of preprocessing options for image augmentation, such as resizing, rotation, and reflection. The image Data Augmenter is used by an augmentedImageDatastore functions to generate batches of augmented images. We expand the number of frames using data augmentation for the images labeled in Section $A$ Types of augmentation are: moving to the left / right direction, enlarging/reducing. Rotation and color tone correction etc., are not carried out. Horizontal movement, enlargement/reduction are performed randomly so that the number of data is increased about five times as many as the original frame number.

\section{G. Validation and Testing}

In this study that there are about 2,200 images available in this dataset. Split the dataset into 3-folds for analysis, i.e., three different algorithms would be trained using different sets of images from the dataset. This type of validation study would provide us a better estimate of our performance compared to the typical hold-out validation method.

We adopt ResNet-50 architecture in this blog as it has proven to be highly effective for various medical imaging applications. One idea of proposed system Replacing the number of classes with 11 according to its categories and Replacing the last layers with new layers

\section{H. Classification of Fetal stage and abnormal:}

After training $\mathrm{CNN}$ resnet50 of proposed system. The system classifies the entered image so that if it has achieved an accuracy of more than $96 \%$ or by choosing the expert, it is automatically stored in the named folder according to the state of fetal health and age within the specified range in the datastore as feedback. 

and Classification of Pregnancy StateUsing Deep Learning Technique,

\section{Results and Discussion}

\section{A. Results:}

After implementing all the steps described in the previous chapter, the system is evaluated, and the final results are discussed. Results obtained from the proposed system automatically during training, validation, and testing process to get accurate results by used cross-validation technics by applying This is to repeat the experiment five times using the values mentioned in the previous chapter, as each investigation resulted in a curve for accuracy and loss. The mean was calculated for accuracy to obtain excellent accuracy due to repetition, training, and verification in each experiment. The three graphic carve are shown in Fig4, Fig5, Fig6, Fig7, Fig8 and The confusion matrix shown in Fig9

The first experiment was Started in split1 from proposed algorithm by selecting the firstfifths of the test's images and the last four fifths for the training. That is the first fold for testing and the second, third, fourth and fifths fold for training. You can see this in Previous Section, Figure 2.

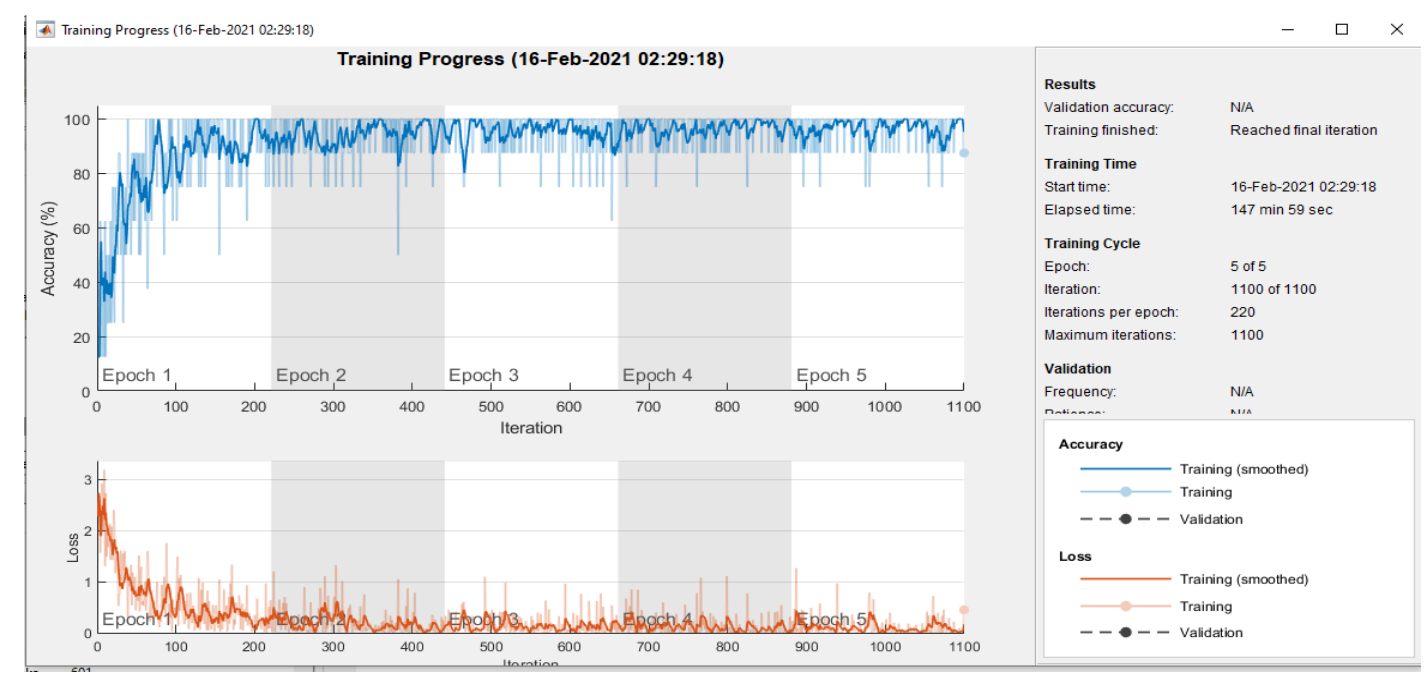

Figure 4: Training Progress in split1

The second experiment was Started in split2 from proposed algorithm by selecting the second fifths of the test's images and the other four fifths for the training. That is the second fold for testing and the first, third, fourth and fifths fold for training. The following results are obtained through the training progress, as shown in Figure 5, where 139 minutes

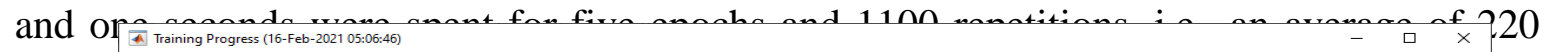
repetit

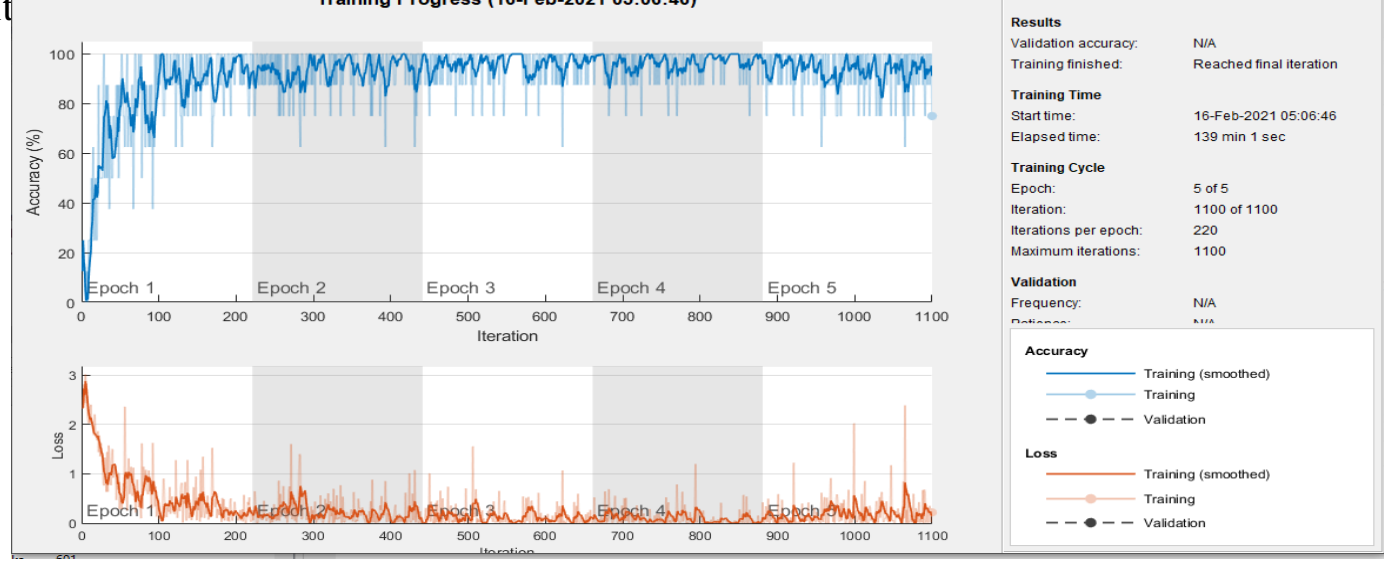

Figure 5: Training Progress in split2 
The third experiment was Started in split3 from proposed algorithm by selecting the third fifths of the test's images and the other four fifths for the training. That is the third fold for testing and the first, second, fourth and fifths fold for training. The following results are obtained through the training progress, as shown in Figure 6, where 142 minutes and 9 seconds were spent for five epochs and 1100 repetitions, i.e., an average of 220 repetitions per epoch.

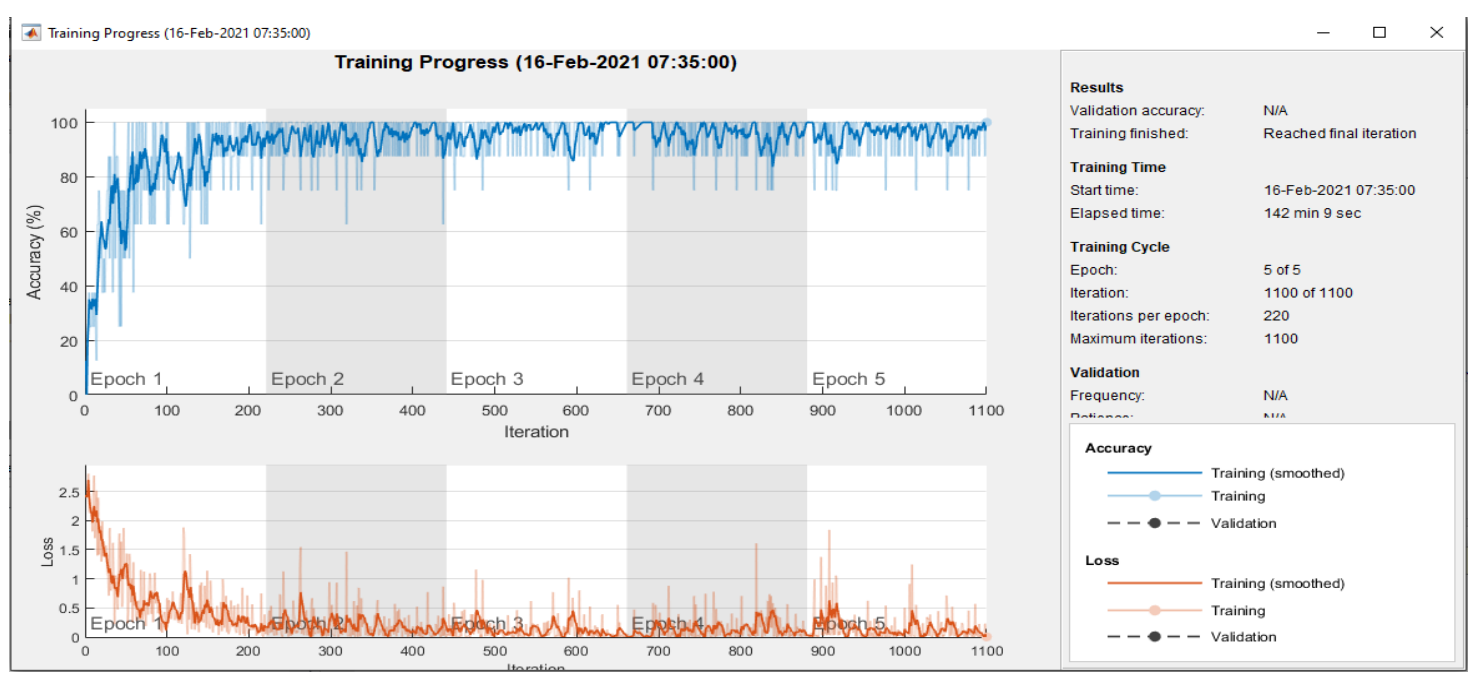

Figure 6: Training Progress in split3

The fourth experiment was Started in split3 from proposed algorithm by selecting the fourth fifths of the test's images and the other four fifths for the training. That is the fourth fold for testing and the first, second, third and fifths fold for training. The following results are obtained through the training progress, as shown in Figure 7, where 139 minutes and 15 seconds were spent for five epochs and 1100 repetitions, i.e., an average of 220 repetitions per epoch.

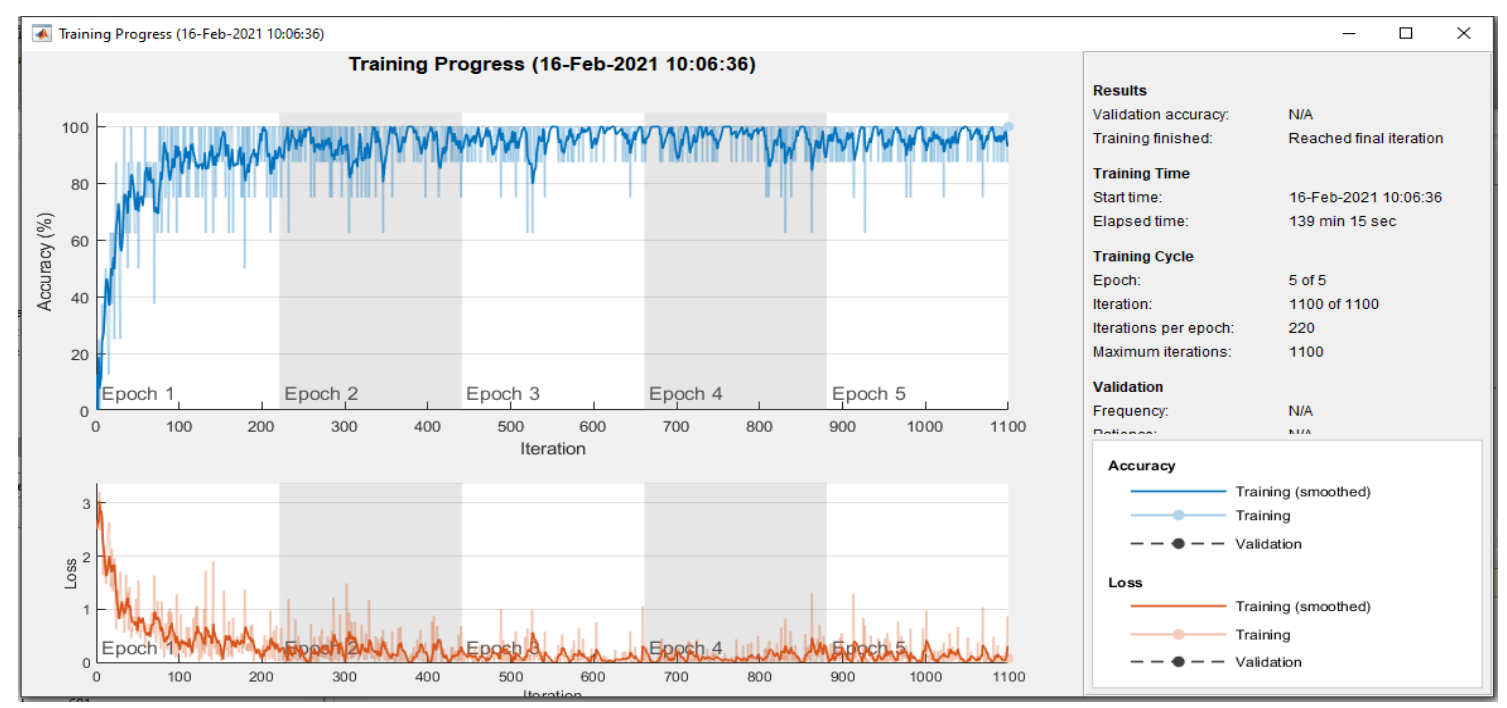

Figure 7: Training Progress in split4 

and Classification of Pregnancy StateUsing Deep Learning Technique,

The fifths experiment was Started in split3 from proposed algorithm by selecting the fife fifths of the test's images and the other four fifths for the training. That is the fifths fold for testing and the first, second, third and fourth fold for training. The following results are obtained through the training progress, as shown in Figure 8, where 139 minutes and 13 seconds were spent for five epochs and 1100 repetitions, i.e., an average of 220 repetitions per epoch

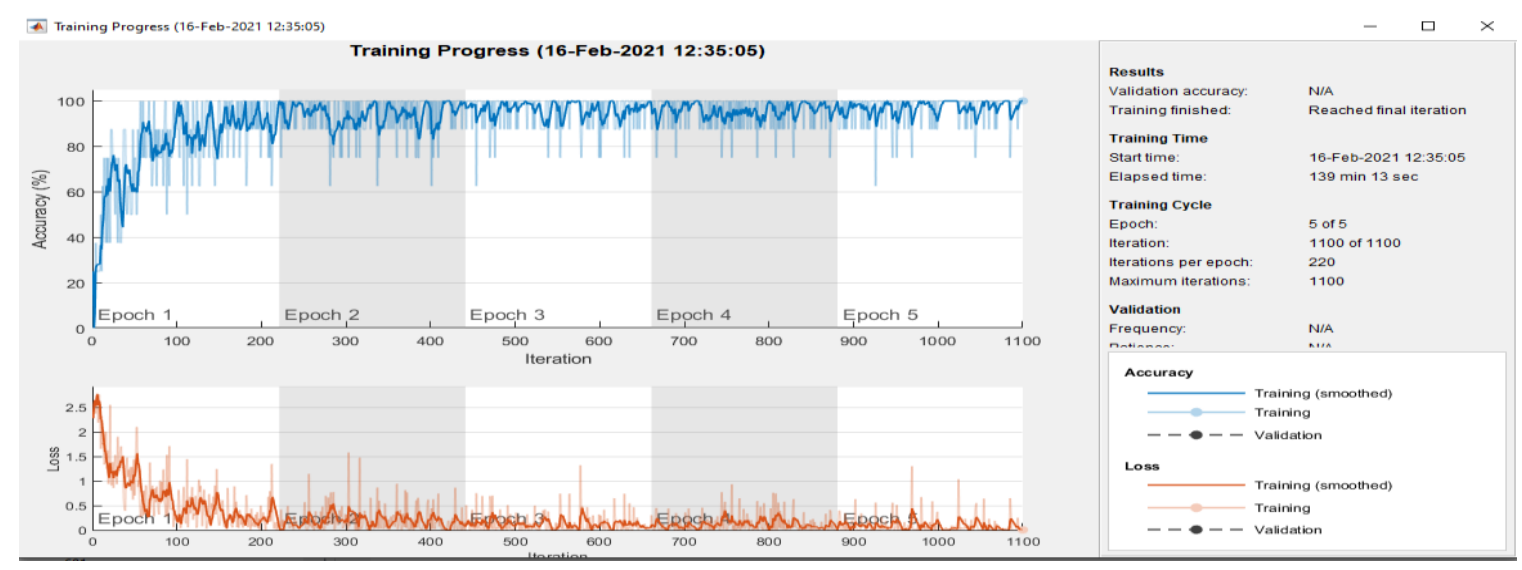

Figure 8: Training Progress in split5

Evaluate the Performance of the Experiments

measured the performance of the proposed algorithm by confusion matrix - This metric also gives a great idea of the performance in duration of accuracy and recall We believe overall accuracy is an excellent pointer as the testing dataset used in this work is consistently spread (in terms of images belonging to each category).as shown in table 5.1. Confusion matrix was achieved with an accuracy of 95.5 for 5 folds to diagnose the fetus's health status during pregnancy for all stages of development shown in Figure 9 below.

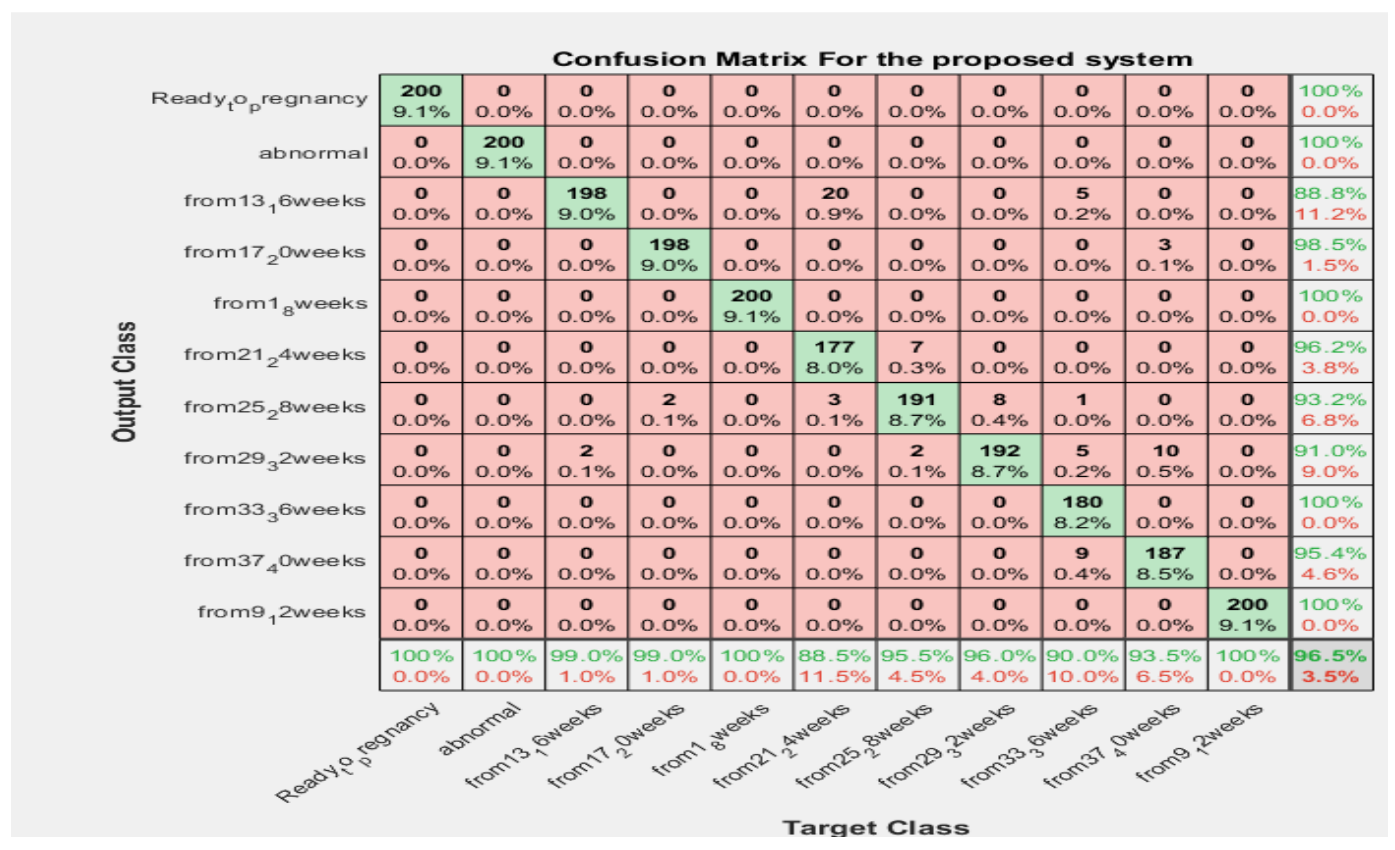

Figure 9: The confusion matrix For the proposed Model 


\section{B. Discussion}

The performance of the pregnancy diagnosis system was evaluated in this study through the confusion matrix presented in the evaluation section to verify its validity. . This study used a new method to detect and classify all stages of fetal development by taking all steps of growth and the readiness of the uterus before pregnancy into account, unlike other studies that focused on a specific part of the fetus's body, such as the heart. Head etc. Or a particular period of growth such as the first trimester of pregnancy or the last one.

This study achieved great success in the initial stages. It failed in something in the advanced stages of fetal growth due to the change of the enlargement of the fetus's organs and the similarity between the species at this stage. And solve this problem by increasing the data and using many reference samples containing all directions after this solution achieved more than $96.5 \%$ accuracy for diagnosing fetal growth status acc. Similarities were found between this study and other studies in detecting the state of the fetus's apparent growth in the use of deep learning. However,this study was distinguished by setting the scope of the study of all stages of development and taking the new images that are used for the test by preserving it as a feedback and taking advantage of its features to enhance training, to be exact. Accurate results and proper diagnosis.

\section{Conclusion}

This paper presented an image processing system to discover and classify the state of fetal health during and before pregnancy. Image acquisition is based on the collection of 2,200 images from Kaggle sources. Pre-processing takes place in the following stages: preparing the datastore, dividing the data into categories, labeled them to access them for training, verification, and testing.

A sum equal to the number of images for each category to be equivalent to the category with the least number of images. To check the readiness of an image from each category, a CNN (resnet50) model was applied as a deep learning application to extract features to detect fetal status and classify the system's input images. Also, the cross-validation technique was used for training with three folds to reach high accuracy of results.

When entering a diagnostic image not included in the data store, it is tested by a specialized expert to accept it and store it in its category as a feedback or to obtain it automatically if the accuracy of its test is more than 95 compared to the manual results, the system results showed excellent efficiency with 97.1\% accuracy in detecting Pregnancy condition.

\section{Acknowledgment}

Authors would like to thank the technicians and staff atthe Laboratories in khartoum state, and Medical Corps Hospital - Omdurman for their assistance and forproviding pathological data. I would also like to thank Dr. Abdeen Rihan Farah Musa for providing health informations. 


\section{References}

Al-Bander, B., Alzahrani, T., Alzahrani, S., Williams, B. M., \& Zheng, Y. (2019). Improving Fetal Head Contour Detection by Object Localisation with Deep Learning. Paper presented at the Annual Conference on Medical Image Understanding and Analysis.

Attallah, O., Sharkas, M. A., \& Gadelkarim, H. (2020). Deep learning techniques for automatic detection of embryonic neurodevelopmental disorders. Diagnostics, 10(1), 27.

Baumgartner, C. F., Kamnitsas, K., Matthew, J., Fletcher, T. P., Smith, S., Koch, L. M., . . Rueckert, D. (2017). SonoNet: real-time detection and localisation of fetal standard scan planes in freehand ultrasound. IEEE transactions on medical imaging, 36(11), 2204-2215.

Deressa, T. D., \& Kadam, K. (2018). Prediction of fetal health state during pregnancy: a survey. Int. J. Comput. Sci. Trends Technol.(IJCST), 6(1).

Diniz, P. H. (2020). Deep Learning Strategies for Ultrasound in Pregnancy. REPRODUCTIVE HEALTH.

Grandjean, G. A., Hossu, G., Banasiak, C., Ciofolo-Veit, C., Raynaud, C., Rouet, L., . . . Beaumont, M. (2019). Optimization of Fetal Biometry With 3D Ultrasound and Image Recognition (EPICEA): protocol for a prospective cross-sectional study. BMJ open, 9(12), e031777.

Ishikawa, G., Xu, R., Ohya, J., \& Iwata, H. (2019). Detecting a Fetus in Ultrasound Images using Grad CAM and Locating the Fetus in the Uterus. Paper presented at the ICPRAM.

Luo, H., Liu, H., Li, K., \& Zhang, B. (2019). Automatic quality assessment for 2D fetal sonographic standard plane based on multi-task learning. arXiv preprint arXiv:1912.05260.

Mu, Y., Feng, K., Yang, Y., \& Wang, J. (2018). Applying deep learning for adverse pregnancy outcome detection with pre-pregnancy health data. Paper presented at the MATEC Web of Conferences.

Qu, R., Xu, G., Ding, C., Jia, W., \& Sun, M. (2019). Deep learning-based methodology for recognition of fetal brain standard scan planes in 2D ultrasound images. IEEE Access, 8, 44443-44451.

Rawat, V., Jain, A., \& Shrimali, V. (2018). Automated techniques for the interpretation of fetal abnormalities: a review. Applied bionics and biomechanics, 2018.

Sobhaninia, Z., Emami, A., Karimi, N., \& Samavi, S. (2020). Localization of fetal head in ultrasound images by multiscale view and deep neural networks. Paper presented at the 2020 25th International Computer Conference, Computer Society of Iran (CSICC).

Sobhaninia, Z., Rafiei, S., Emami, A., Karimi, N., Najarian, K., Samavi, S., \& Soroushmehr, S. R. (2019). Fetal ultrasound image segmentation for measuring biometric parameters using multi-task deep learning. Paper presented at the 2019 41st Annual International Conference of the IEEE Engineering in Medicine and Biology Society (EMBC).

Tahir, M., Badriyah, T., \& Syarif, I. (2018). Classification Algorithms of Maternal Risk Detection For Preeclampsia With Hypertension During Pregnancy Using Particle Swarm Optimization. EMITTER International Journal of Engineering Technology, 6(2), 236-253.

Tran, D., Cooke, S., Illingworth, P., \& Gardner, D. (2019). Deep learning as a predictive tool for fetal heart pregnancy following time-lapse incubation and blastocyst transfer. Human Reproduction, 34(6), 1011-1018.

Wang, S., Hua, Y., Cao, Y., Song, T., Xue, Z., Gong, X., . . Guan, H. (2018). Deep learning based fetal middle cerebral artery segmentation in large-scale ultrasound images. Paper presented at the 2018 IEEE International Conference on Bioinformatics and Biomedicine (BIBM).

Zhang, J., Petitjean, C., Yger, F., \& Ainouz, S. (2020). Explainability for regression CNN in fetal head circumference estimation from ultrasound images. In Interpretable and Annotation-Efficient Learning for Medical Image Computing (pp. 73-82): Springer. 